\title{
Comparação da Eficiência dos Biocombustíveis e Gás Natural Usados em Fornalhas para Secagem de Grãos Utilizando a Modelagem Matemática
}

C.J. AUTH, A.P. SPILIMBERGO, V.G. KRIOUKOV, Universidade Regional do

Noroeste do Estado do Rio Grande do Sul (UNIJUÍ), Departamento de Física, Estatística e Matemática e Departamento de Tecnologia, Cx.P. 560, 98700-000 Ijuí, RS, Brasil.

\begin{abstract}
Resumo. Fornalhas com fluxos forçados são usadas amplamente na Região Sul, para realizar a secagem de diferentes tipos de grãos. Tradicionalmente a biomassa é utilizada como combustível, mas a chegada na região de uma tubulação de gás natural, possibilita utilizar o gás em uma fornalha típica. O presente trabalho tem por objetivo realizar a comparação da eficiência dos combustíveis de biomassa (lenha de eucalipto, casca de arroz e bagaço de cana) e do gás, usando a abordagem da modelagem matemática. Para isso o modelo desenvolvido anteriormente (Dalepiane et al. [3]) foi modificado nos aspectos: fluxo de calor por radiação, aquecimento das paredes e perdas hidráulicas. As simulações numéricas realizadas mostraram algumas vantagens no caso da utilização do gás como combustível: maior rendimento; baixas temperaturas das paredes na zona da grelha; maiores temperaturas dos produtos de combustão, que contribui para a queima mais completa do combustível.
\end{abstract}

\section{Introdução}

No sul do Brasil, combustíveis de biomassa são utilizados largamente em fornalhas destinadas à secagem de diferentes espécies de grãos. Um esquema típico destas fornalhas está apresentado na Figura 1. Mas na utilização da biomassa, além da produção de resíduos indesejáveis (ver [10]), existe a possibilidade de comprometimento dos recursos naturais (como no caso da lenha). Por outro lado revela-se como uma perspectiva, a utilização do gás como fonte de energia nos processos de secagem, permitindo assim controlar a combustão, tendo um alto poder calorífico, além de contribuir para a preservação dos recursos naturais e não gerar resíduos. É por isso que a realização da análise comparativa das características operacionais de uma fornalha, utilizando biocombustíveis ou gás torna-se um problema interessante.

Via de regra a determinação das características de uma fornalha é feita por ensaios experimentais, mas a abordagem da modelagem matemática se mostra uma ferramenta eficiente e barata para determiná-las (ver $[6,9,10,12,13])$. Um exemplo se encontra em Dalepiane et al. [3] para a fornalha apresentada na Figura 1, quando, 
com base em um modelo elaborado pelos referidos autores, foram determinadas em função do tempo características como: rendimento, perdas de pressão, aquecimento das paredes, temperatura na grelha, etc., para um único tipo de combustível. Portanto, levando em conta o mesmo tipo de fornalha, este trabalho visa determinar as suas principais características operacionais, considerando outros combustíveis de biomassa (lenha de eucalipto, bagaço de cana e casca de arroz) e gás.

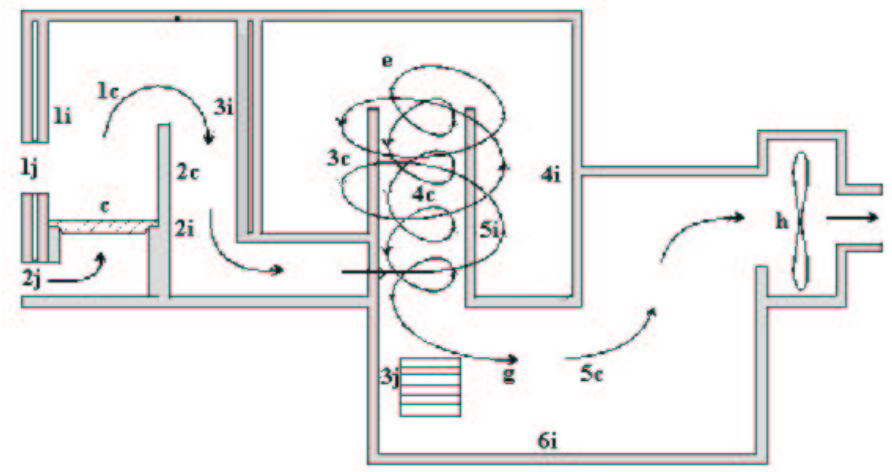

Figura 1: Esquema de uma fornalha para secagem de grãos.

A estrutura de uma fornalha industrial da Figura 1 é composta pelos fragmentos: $1 \mathrm{j}$ - janela para abastecer a fornalha com combustível; $2 \mathrm{j}$ - janela para admissão de ar primário; $3 \mathrm{j}$ - janela para admissão controlada de ar secundário; c - grelha; 1c, 2c, 3c, 4c, 5c - trajetos; 1i, 2i, 3i, 4i, 5i, 6i - paredes; g - zona de mescla dos produtos de combustão com ar secudário; e - ciclone e $\mathrm{h}$ - ventilador. O seu princípio de trabalho é simples: o combustível, jogado por cima para a grelha, queima com o ar que flui da janela $2 \mathrm{j}$. Então, na grelha ficam os carvões queimados e na entrada do trajeto 1c formam-se os produtos de combustão de alta temperatura. Os produtos de combustão passando pelos trajetos aquecem as paredes. Um efeito importante é causado pelos carvões na grelha, que irradiam um grande fluxo de calor nas paredes da fornalha, provocando uma mais alta temperatura das paredes $\left(T_{p}\right)$ no primeiro trajeto, do que a temperatura dos produtos de combustão na grelha $\left(T_{g r}\right)$. Na entrada do trajeto 3c (ciclone) forma-se o fluxo com redemoinho para separar as partículas sólidas do gás. Na saída do trajeto 4c, o gás mescla-se com o ar secundário para resfriar-se até $T_{s}$ (aproximadamente de $1300 \mathrm{~K}$ até $390 \mathrm{~K}$ ) e, logo após, pelo trajeto $5 \mathrm{c}$, esta mistura entra no ventilador que a puxa para o secador de grãos.

Durante o funcionamento da fornalha os parâmetros de controle: consumo de combustível $\left(G_{c}\right)$, temperatura na saída $\left(T_{s}\right)$ e potência do ventilador $(W)$ são mantidos aproximadamente constantes. Mas apesar disso observa-se o regime transiente, pois no início do seu funcionamento a temperatura das paredes é a mesma do meio ambiente e o fluxo de calor para elas é grande. A medida que a temperatura das paredes aumenta, a transferência de calor para elas diminui. Como resultado, na 
saída do ciclone o fluxo possui maior energia, e é necessário então (para manter $T_{s}=$ const.) um aumento na vazão do ar secundário, o que provoca o crescimento de $G_{s}$ (vazão total de "ar quente") e o caráter transiente do regime operacional. grossas.

\section{Processos em Fornalhas e Modelo Matemático}

O modelo matemático da fornalha (Figura 1), considera os fenômenos: da combustão na grelha; dos fluxos dos produtos de combustão pelos trajetos, incluindo o ciclone, considerando o atrito e a transferência de calor; das perdas locais de pressão; da mescla dos produtos de combustão com ar secundário; da transferência de calor por radiação e convecção; do aquecimento não-estacionário das paredes e da "adaptação da vazão" através do ventilador. Além disso leva em conta algumas hipóteses como: combustão em equilíbrio químico (ver [1]); distribuição unidimensional de calor nas paredes; distribuição uniforme de calor por radiação, nas paredes 1, 2 e 3; consumo do combustível e temperatura $T_{s}$ constantes; etc. O modelo matemático é composto por diferentes submodelos, a seguir.

1. Submodelo de combustão. Diferentemente de Dalepiane et al. [3], ele basea-se na equação da energia, encontrada em Stehlik et al. [12], com a adição de termos correspondentes ao fluxo de calor por radiação:

$$
T_{g r}=T_{a d}-\frac{Q_{g r}+Q_{1,2}}{\bar{C}_{p} \cdot G_{p c}}-\frac{\Delta H_{b} \cdot \phi}{\bar{C}_{p} \cdot\left(\alpha_{o x}^{\prime} \cdot k_{m}^{o}+1\right)},
$$

onde $\quad Q_{g r}=A_{g r} K_{r d} \sigma \epsilon_{g r}\left(\left[\frac{T_{a d}}{100}\right]^{4}-\left[\frac{T_{p 1}}{100}\right]^{4}\right) \quad$ e $\quad Q_{1,2}=A_{1,2} \sigma\left(\left[\frac{T_{p 1}}{100}\right]^{4}-\left[\frac{T_{p 2}}{100}\right]^{4}\right)$, sendo: $G_{p c}$ e $\bar{C}_{p}$ - vazão e calor específico médio dos produtos de combustão nos trajetos 1c e 2c, respectivamente; $\phi$ - coeficiente das perdas de energia na grelha $\left(\phi \approx 0,02 \ldots 0,05^{[14]}\right) ; \Delta H_{b}$ - poder calorífico baixo; $T_{a d}$ - temperatura adiabática; $\alpha_{o x}^{\prime}$ - coeficiente de excesso do oxidante na grelha e nos quatro primeiros canais; $k_{m}^{o}$ - razão mássica estequiométrica entre o oxidante e o combustível; $Q_{g r}$ e $Q_{1,2}$ - fluxo de calor por radiação da grelha e do trajeto $1 \mathrm{c}$ para $2 \mathrm{c}$, respectivamente; $A_{g r}$ - área da grelha; $K_{r d}$ - coeficiente de identificação; $\sigma$ - constante de Stefan-Boltzmann; $\epsilon_{g r}$ - emissividade do carvão na grelha; $T_{p 1}$ e $T_{p 2}$ - temperaturas médias das paredes nos trajetos $1 \mathrm{c}$ e $2 \mathrm{c}$, respectivamente; $A_{1,2}$ - área da passagem entre os trajetos $1 \mathrm{c}$ e 2 c.

2. Submodelo de transferência de calor nas paredes. Este submodelo inclui a equação de transferência do calor dentro de cada parede ([11]) considerando, diferentemente de Dalepiane et al. [3], a dependência $\lambda_{p}=f\left(T_{p}\right)$ e a separação dos fluxos de calor por convecção e por radiação:

$$
\frac{\partial T_{p i}}{\partial \tau}=\frac{1}{\rho_{p i} . C^{p i}} \frac{\partial}{\partial y_{p i}}\left(\lambda_{p i} \frac{\partial T_{p i}}{\partial y_{p i}}\right)
$$

com as condições de contorno: 


$$
h_{k}\left(T-T_{p i}^{g}\right)+q_{i}^{r d}=-\lambda_{p i}\left(\frac{\partial T_{p i}}{\partial y_{p i}}\right)_{g p} \text { e } \quad h_{a}\left(T_{p i}^{a}-T_{a}\right)=-\lambda_{p i}\left(\frac{\partial T_{p i}}{\partial y_{p i}}\right)_{a p},
$$

onde $\quad q_{1}^{r d}=\frac{Q_{g r}}{B_{1} l_{e f 1}} ; \quad q_{2}^{r d}=\frac{Q_{g r}}{B_{1} l_{e f 1}} ; \quad q_{3}^{r d}=\frac{Q_{1,2}}{B_{2} l_{e f 2}} ; \quad q_{i}^{r d}=0 ; \quad i=4,5,6, \quad$ sendo: $\tau$ - tempo; $T_{p i}$ e $y_{p i}$ - temperatura e coordenada corrente pela espessura, respectivamente; $\rho_{p i}, C^{p i}$ e $\lambda_{p i}$ - densidade, calor específico e condutibilidade térmica do material da parede, respectivamente; $h_{k}$ e $h_{a}$ - coeficientes de transferência de calor por convecção dos lados quente e frio, respectivamente; $q_{i}^{r d}$ - fluxo de calor por radiação; $i$ - número da parede; $B_{k}$ e $l_{e f k}$ perímetro e comprimento efetivo do k-ésimo trajeto, respectivamente; $T_{a}$ - temperatura atmosférica.

3. Submodelo dos fluxos pelos trajetos. Ele é apresentado pelas equações unidimensionais transientes de gás compressível, considerando o atrito e a transferência de calor $([11])$

$$
\begin{gathered}
\frac{d T_{k}}{d x}=-\frac{h_{k} \cdot B_{k}\left(T_{k}-T_{p}(\tau)\right)}{G_{k} \cdot C_{p k}}=-f_{T_{k}}, \\
\frac{d P_{k}}{d x}=\left(\frac{f_{T_{k}}}{T_{k}}-\frac{\xi_{k}}{2 D_{e f k}}\right) /\left(\frac{A_{e f k}^{2} \cdot P_{k}}{G_{k}^{2} \cdot R \cdot T_{k}}-\frac{1}{P_{k}}\right),
\end{gathered}
$$

onde $G_{i}=G_{p c}(i=1 \ldots 4) ; G_{i}=G_{s}(i=5) ; x$ - coordenada ao longo do trajeto; $R$ - constante do gás, $P_{k}$ - pressão; $A_{e f k}$ - área efetiva, $\xi_{k}$ - fator do atrito; $D_{e f k}$ diâmetro efetivo. O número de Nusselt em cada trajeto é determinado por:

$$
N u_{k}=0,145 \operatorname{Re}_{k}^{0,72} \operatorname{Pr}_{k}^{0,33}\left(\frac{L_{t}}{D_{\text {efk }}}\right)^{0,6} \exp \left(1-\frac{L_{t}}{D_{\text {efk }}}\right),
$$

onde $L_{t}$ é a espessura de um tijolo, $R e$ e $\operatorname{Pr}$ os número de Reynolds e Prandtl. A equação (2.4), retirada de Gortishev e Olimpiev [4], é mais adequada pois reflete a transferência de calor na parede de tijolos. O fator de atrito (usando a analogia de Reynolds, ver [11]) é determinado pela relação:

$$
\xi_{k}=\frac{8 N u_{k} P r_{k}^{2 / 3}}{R e_{k}} .
$$

Os fluxos com redemoinho (trajetos 3c e 4c) são descritos pelas equações (2.2) e (2.3) com a correção ([8]) dos coeficientes $\xi_{k}$ e $h_{k}$ :

$$
h_{k}=h_{k}^{o}\left(1+1,15 \Phi^{1,06}\right) \text {, }
$$

onde $h_{k}^{o}$ é o coeficiente da transferência de calor sem redemoinho e $\Phi$ é a taxa de torção que é determinada, para o ciclone externo e interno, respectivamente por

$$
\Phi_{e x t}=\frac{2 \pi R_{e x}^{2}\left(1-X^{3}\right)}{3 a b} \quad \text { e } \quad \Phi_{i n t}=\frac{2 \pi R_{e x}^{2}\left(1+X+X^{2}\right)}{3 a b(1+X)},
$$


onde $X=\left(r_{o}+\delta\right) / R_{e x} ; r_{o}$ e $R_{e x}$ - raios dos trajetos do ciclone interno e externo e $\delta$ - espessura da parede 5 i.

4. Perdas locais de pressão entre os canais. Foram determinadas pelas fórmulas tradicionais apresentadas em Lencastre [7].

5. Submodelo da mescla dos produtos de combustão com ar secundário. Baseado nas equações encontrada em Alemassov et al. [1]:

$$
\begin{gathered}
I_{p c}\left(\alpha_{o x}^{\prime}, T_{c i}\right)\left(1+\alpha_{o x}^{\prime} k_{m}^{o}\right)+I_{o x}\left(\alpha_{o x}^{s}-\alpha_{o x}^{\prime}\right)=I_{p c}\left(\alpha_{o x}^{s}, T_{s}\right)\left(1+\alpha_{o x}^{s} k_{m}^{o}\right), \\
G_{o x}^{\prime \prime}=\left(\alpha_{o x}^{s}-\alpha_{o x}^{\prime}\right) \cdot k_{m}^{o} \cdot G_{c} \quad \text { e } \quad G_{s}=\left(\alpha_{o x}^{s} \cdot k_{m}^{o}+1\right) \cdot G_{c},
\end{gathered}
$$

onde $I_{p c}\left(\alpha_{o x}^{\prime}, T_{c i}\right)$ e $I_{p c}\left(\alpha_{o x}^{s}, T_{s}\right)$ - entalpias dos produtos de combustão com temperatura no ciclone $\left(T_{c i}\right)$ e com temperatura $T_{s}$, respectivamente; $\alpha_{o x}^{s}$ - coeficiente de excesso do oxidante (ar) na saída; $I_{o x}$ - entalpia do ar; $G_{o x}^{\prime \prime}$ - vazão do ar secundário. 6. Equação da adaptação do fluxo. Realiza o ajuste no ponto de trabalho na característica do ventilador $([3])$ :

$$
\frac{G_{s}}{\rho_{s} Q_{n m}}=W \cdot \sum_{q=0}^{3} a_{q} \cdot\left(\left(\sum_{k} \Delta P_{k}+\sum_{k} \Delta P_{k}^{m}\right) / \Delta P^{n m} \cdot W\right)^{q},
$$

onde $Q_{n m}$ e $\Delta P_{k}^{n m}$ correspondem a vazão e a diferença de pressão total no ventilador no regime nominal, os valores $a_{q}$ são coeficientes de aproximação das características do ventilador e $\Delta P_{k}^{m}$ as perdas menores de pressão.

Como em Dalepiane et al. [3] as incógnitas no modelo modificado são as características operacionais da fornalha: $G_{S}(\tau), G_{o x}^{\prime}(\tau)$ (vazão do ar pela janela 2), $\Delta P_{s}(\tau), \theta(\tau), T_{g r}(\tau)$, e as evoluções de $T_{p i}, \Delta P_{k}, \Delta P_{k}^{m}$ e $\alpha_{o x}^{s}$ pelo tempo. São conhecidos $G_{c}, T_{s}, W$, tamanhos geométricos dos trajetos e das paredes, característica energéticas do material das paredes $\left(\lambda_{p}, C^{p}, \rho_{p}\right)$, características energéticas e a composição do combustível, temperatura do meio ambiente e características de trabalho do ventilador $\left(Q=f\left(W, \Delta P_{s}\right)\right)$.

Em princípio o algoritmo do cálculo é constituído pelas etapas a seguir.

1. Cálculos preliminares e escolha de $G_{o x}^{\prime}$.

2. Cálculo das características de combustão utilizando interpolações.

3. Determinação de $\Delta T_{k}, \Delta P_{k}$ e $\Delta P_{k}^{m}$ nos trajetos $\mathrm{k}=1 \ldots 4$.

4. Cálculo das características da mistura com ar secundário: equação (2.5).

5. Determinação de $\Delta T_{k}, \Delta P_{k}$ e $\Delta P_{k}^{m}$ no trajeto $\mathrm{k}=5$ e do erro na equação (2.6).

6. Comparação: se o erro na equação (2.6) for maior do que o erro admissível é realizada a correção de $G_{o x}^{\prime}$ e ocorre a volta ao item 2 ; caso contrário é realizado o cálculo da distribuição das temperaturas nas paredes i $=1 \ldots 6$ (equação (2.1)).

7. Se $\tau<\tau_{f}\left(\tau_{f}\right.$ - tempo de funcionamento da fornalha) retornar ao item 2, caso contrário terminar o cálculo.

O fragmento da combustão é o de maior imporância para o presente trabalho e utiliza no algoritmo, a matriz (Tabela I) das propriedades dos produtos de combustão, com interpolação destas propriedades para pontos intermediários. Nesta tabela, na primeira coluna estão apresentados os valores de $\alpha_{o x}^{\prime}$ e em cada linha 
as propriedades correspondentes: $C_{p}(\mathrm{~kJ} / \mathrm{kg} . \mathrm{K})$ - calor específico, $\eta\left(\mathrm{N} . \mathrm{s} / \mathrm{m}^{2}\right)$ - viscosidade, $\lambda(\mathrm{W} / \mathrm{m} . \mathrm{K})$ - condutibilidade térmica, $I(\mathrm{~kJ} / \mathrm{kg})$ - entalpia e $\mu(\mathrm{kg} / \mathrm{kmol})$ - massa molecular média, que são utilizadas em outros fragmentos do modelo matemático da fornalha e são necessárias para determinar o fluxo de calor nas paredes, a velocidade nos canais, a variação de pressão, etc, nos trajetos. O intervalo de alteração de $\alpha_{o x}^{\prime}$ foi de 0,5 a 10 com $\Delta \alpha_{o x}^{\prime}=0,25$.

Tabela I - Fragmento da matriz das propriedades dos produtos de combustão na grelha, para a lenha de eucalipto (com umidade de $20 \%$ )

\begin{tabular}{|c|c|c|c|c|c|c|}
\hline$\alpha_{o x}^{\prime}$ & $T$ & $I$ & $\mu$ & $C_{p}$ & $\eta$ & $\lambda$ \\
\hline 0,5 & 1340 & -2390 & 25,4 & 1,60 & $5,4810^{-5}$ & 0,126 \\
\hline 0,75 & 1800 & -1770 & 27,5 & 1,52 & $6,8810^{-5}$ & 0,15 \\
\hline$\ldots$ & $\ldots$ & $\ldots$ & $\ldots$ & $\ldots$ & $\ldots$ & $\ldots$ \\
\hline 9,75 & 586 & -172 & 29 & 1,12 & $3,7110^{-5}$ & 0,06 \\
\hline 10,0 & 580 & -168 & 29 & 1,12 & $3,7110^{-5}$ & 0,06 \\
\hline
\end{tabular}

Esta matriz (Tabela I) é calculada preliminarmente e é apresentada no aplicativo principal (FORNIJ - que é a modificação do aplicativo FORN ([3]), como um dos arquivos de dados iniciais, onde, para cada combustível, é necessário gerar uma matriz específica com a ajuda do software ADTIJUI (Auth et al. [2]), que executa cálculos para sistemas no estado de equilíbrio químico.

\section{Pesquisas Numéricas}

A Tabela II apresenta as características geométricas nas simulações numéricas dos processos em fornalha com fluxo forçado (Figura 1). Onde: $A_{e f}, L_{e f}$ - são áreas e comprimentos dos trajetos, $W=0,95, \phi=0,03, k_{r d}=0,3, T_{s}=400 K, G_{s}^{e s}=$ $4,40(\mathrm{~kg} / \mathrm{s})$ - fluxo mássico na saída do ventilador no regime estacionário, $A_{p}$ e $\delta_{p}$ área efetiva e espessura da parede. As paredes 1i e 3i são multicamadas construídas de tijolos refratários $(10 \mathrm{~cm})$, fibra de vidro $(10 \mathrm{~cm})$ e tijolos de argila $(10 \mathrm{~cm})$.

Tabela II - Características geométricas da fornalha

\begin{tabular}{|c|c|c|c|c|c|c|}
\hline Trajeto & $1 c$ & $2 c$ & $3 c$ & $4 c$ & $5 c$ & grelha \\
\hline$A_{e f}\left(m^{2}\right)$ & 0,7 & 0,7 & 0,67 & 0,20 & 15 & 0,7 \\
\hline$L_{e f}(m)$ & 1,2 & 1,2 & 1,0 & 1,0 & 2,0 & - \\
\hline Parede & $1 \mathrm{i}$ & $2 \mathrm{i}$ & $3 \mathrm{i}$ & $4 \mathrm{i}$ & $5 \mathrm{i}$ & $6 \mathrm{i}$ \\
\hline$A_{p}\left(m^{2}\right)$ & 3,51 & 1,89 & 3,10 & 4,84 & 1,44 & 9,20 \\
\hline$\delta_{p}(m)$ & 0,3 & 0,1 & 0,3 & 0,2 & 0,1 & 0,1 \\
\hline
\end{tabular}

Nas simulações numéricas as informações foram obtidas: sobre a biomassa, de Jenkins [5] e sobre o gás da empresa Liquigás e se encontram na Tabela III. 
Tabela III - Características energéticas e composição (\% mássico) dos combustíveis $\left(*-20 \%\right.$ de umidade; $\Delta H_{a}$ em $\mathrm{MJ} / \mathrm{kg}$ )

\begin{tabular}{|c|c|c|c|c|c|c|c|c|c|}
\hline Nome & $\Delta H_{a}$ & $C$ & $H$ & $O$ & $N$ & Cinza & $k_{m}^{o}$ & $\alpha_{o x}^{i d}$ & $P_{f}^{i d}$ \\
\hline Lenha* & 19,23 & 48,18 & 5,92 & 44,78 & 0,60 & 1,12 & 5,6 & 27,5 & 125,6 \\
\hline Bagaço* & 17,33 & 44,8 & 5,35 & 44,77 & 0,51 & 9,79 & 5,27 & 27,7 & 110,4 \\
\hline Casca* & 16,14 & 40,96 & 4,3 & 45,64 & 0,54 & 18,34 & 4,63 & 29,5 & 118,2 \\
\hline Gás & 49,06 & 30,74 & 69,26 & - & - & - & 15,04 & 28,5 & 429,6 \\
\hline
\end{tabular}

O rendimento da fornalha $(\theta)$ é determinado como a razão das vazões do "ar quente"na saída da fornalha:

$$
\theta=\frac{G_{s}}{G_{s}^{i d}}=\frac{\alpha_{o x}^{s} k_{m}^{o}+1}{\alpha_{o x}^{i d} k_{m}^{o}+1}
$$

onde o valor $G_{s}^{i d}$ é uma vazão virtual quando todos os valores de $h_{k}$ são nulos. O valor $\alpha_{o x}^{i d}$ depende somente do tipo de combustível e da $T_{s}$ e determina-se pelo modelo de equilíbrio químico. Com base em $\alpha_{o x}^{i d}$ e $\alpha_{o x}^{s}$ determinam-se as produtividades ideal e real da fornalha:

$$
P_{f}^{i d}=\frac{G_{s}^{i d}}{G_{c}}=\frac{G_{c}\left(\alpha_{o x}^{i d} k_{m}^{o}+1\right)}{G_{c}}=\alpha_{o x}^{i d} k_{m}^{o}+1 \quad \text { e } \quad P_{f}=\alpha_{o x}^{s} k_{m}^{o}+1,
$$

e é evidente que o gás possui $P_{f}^{i d}$ muito maior do que a biomassa, devido ao alto valor do seu poder calorífico.

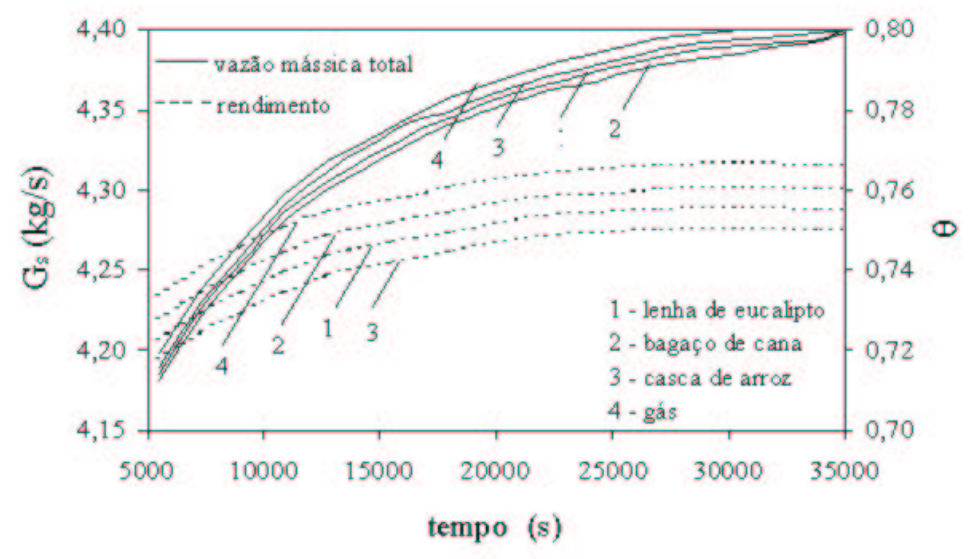

Figura 2: Evolução das características $G_{s}$ e $\theta$ para os combustíveis pesquisados

Na Figura 2 estão mostradas as evoluções da vazão $\left(G_{s}\right)$ e rendimento $(\theta)$ durante o funcionamento da fornalha. Para comparação foi determinado o valor da vazão total à ser atingida igualmente no regime estacionário para os diferentes combustíveis $\left(G_{s}^{e s}=4,40 \mathrm{~kg} / \mathrm{s}\right)$. E naturalmente os valores $G_{c}$ são muito maiores para 
a biomassa do que para o gás, sendo $G_{c}$ (eucalipto $)=0,047 \mathrm{~kg} / \mathrm{s} ; G_{c}($ casca $)=0,054$ $\mathrm{kg} / \mathrm{s} ; G_{c}($ bagaço $)=0,049 \mathrm{~kg} / \mathrm{s}$ e $G_{c}$ (gás) $=0,0132 \mathrm{~kg} / \mathrm{s}$. Devido as perdas de calor a fornalha não possui a produtividade ideal, tendo o rendimento em torno de $\theta=0,72 \ldots 0,77$ para os combustíveis pesquisados.

De acordo com o gráfico observa-se que $G_{s}$ aumenta com o passar do tempo, devido a diminuição das perdas de calor nas paredes, na medida em que entra no regime estacionário e pelo mesmo motivo os rendimentos aumentam para todos os combustíveis. Os biocombustíveis possuem menores rendimentos pois na sua queima são gerados os carvões que irradiam um grande fluxo de calor (mais do que $80 \%$ da transferência total). Para os combustíveis de biomassa os rendimentos mostram as dependências invertidas das temperaturas adiabáticas, para $\alpha_{o x}^{\prime}=1$. A menor $T_{a d}\left(\alpha_{o x}^{\prime}=1\right)$, caso do eucalipto, corresponde ao maior valor $\theta$.

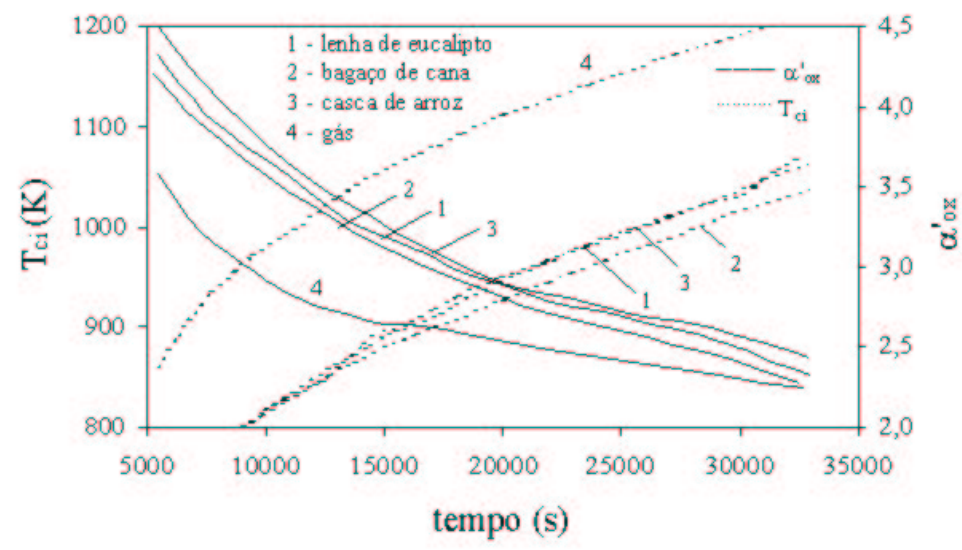

Figura 3: Alteração de $\alpha_{o x}^{\prime}$ e da $T_{c i}$ em função do tempo

Na Figura 3 estão apresentadas as evoluções da temperatura no ciclone $\left(T_{c i}\right)$ e $\alpha_{o x}^{\prime}$ para os diferentes combustíveis. É evidente que $T_{c i}($ gás $)>T_{c i}($ bio $)$ com $\Delta T \approx$ $200 K$ ( $T_{c i}$ (bio) são praticamente iguais). A diferença $\Delta T$ é explicada pela diferença $\operatorname{dos} \alpha_{o x}^{\prime}$, que é menor para o caso do gás. Na zona $\alpha_{o x}^{\prime}>1$, o menor $\alpha_{o x}^{\prime}$ corresponde a maior $T_{a d}$. Este fato é explicado pela necessidade de adaptação dos consumos e pelo alto valor de $k_{m}^{o}$ para o gás. A diminuição do $\alpha_{o x}^{\prime}$ (como regra geral) pelo tempo é condicionada pelo aumento da vazão $\left(G_{s}\right)$, como resultado diminui $\Delta P_{s}$ (na característica do ventilador existe a dependência $G_{s} \sim 1 / P_{s}$ ) e, como conseqüência, diminui $G_{p c}^{\prime}$ e $\alpha_{o x}^{\prime}$ (pois $G_{c}$ é constante).

Na Fig. 4 são mostradas as evoluções das temperaturas nas superfícies das paredes $\left(T_{p 1}\right.$ e $\left.T_{p 3}\right)$. Observa-se uma diferença notável $\left(\Delta T_{p} \approx 200 K\right)$ devido a geração de grande fluxo de calor por radiação nas paredes do primeiro canal (no caso da biomassa). No caso do gás esta radiação é muito menor, devido a isso $T_{p 1}$ (gás) $<T_{p 1}$ (bio). O mesmo efeito se observa no canal $2, T_{p 3}$ (gás) $<T_{p 3}($ bio $)$, no qual penetra uma parcela de radiação do primeiro canal. Mas a diferença $\Delta T_{p 3}$ é pequena, $\Delta T_{p 3}<40 \mathrm{~K}$, pois a intensidade da radiação é menor do que no canal 1 . As temperaturas das superfícies evoluem aumentando até atingir o regime estacionário 


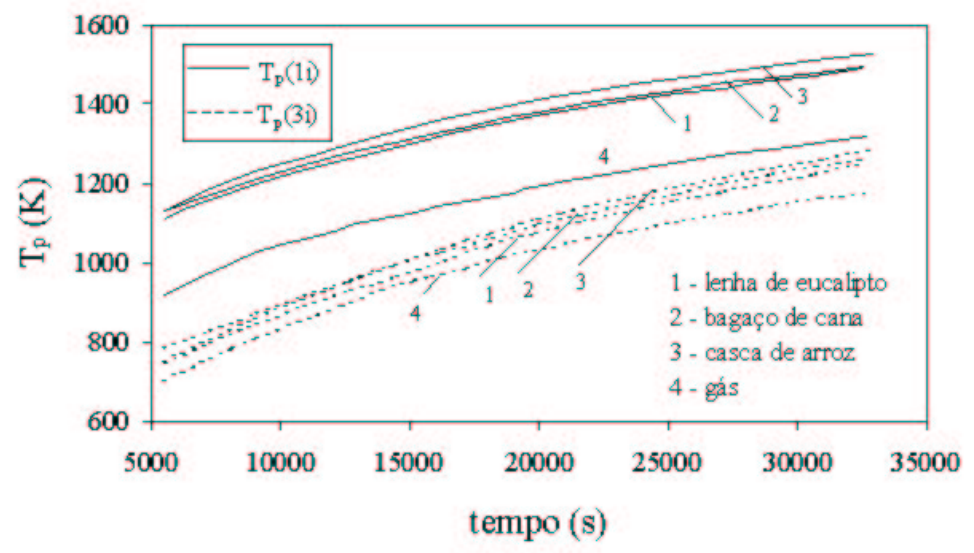

Figura 4: Variação das temperaturas nas paredes pelo tempo

de funcionamento da fornalha, $T_{p 1} \approx 1500 \mathrm{~K}$ e $T_{p 3} \approx 1100 \mathrm{~K}$ para a biomassa $\mathrm{e}$ $T_{p 1} \approx 1200 K$ e $T_{p 3} \approx 1000 K$ para o gás.

\section{Conclusões}

Neste trabalho foi realizada uma análise comparativa das características operacionais de uma fornalha para a secagem de grãos. Nas pesquisas numéricas foram envolvidos os combustíveis de biomassa (lenha de eucalipto, bagaço de cana e casca de arroz) e gás. Em relação aos resultados pode-se fazer as seguintes considerações: - a casca de arroz apresenta menor rendimento e menor produtividade, sendo que as produtividades dos combustíveis de biomassa são próximas. O gás é o que apresenta maior produtividade (mais de 3 vezes);

- o regime estacionário é estabelecido ao mesmo tempo para todos os combustíveis; - as temperaturas nas paredes no primeiro e segundo canal são maiores para os combustíveis de biomassa, se comparadas com $T_{p 1}$ e $T_{p 3}$ no caso do gás. Isto ocorre devido aos fluxos de calor por radiação gerados nos carvões da biomassa;

- com o passar do tempo o coeficiente $\alpha_{o x}^{\prime}$ diminui enquanto que a temperatura no ciclone aumenta para todos os combustíveis; o coeficiente $\alpha_{o x}^{\prime}$ é mais baixo para o gás (mas é necessário um cuidado especial para o não aparecimento do regime anormal ([3]) de funcionamento).

Abstract. Furnaces with forced flows are used widely in the South Region, to carry through the drying of different types of grains. Traditionally the biomassa is used as fuel, but the coming in the region of a natural gas tubing, makes possible to use the gas in a typical furnace. The present work has for objective to carry through the comparison of the efficiency of the biomass fuels (eucalyptus, rice hulls and sugarcane bagasse) and of the gas, using the boarding of the mathematical modeling. For this the developed model previously (Dalepiane et al. [3]) was modified in the aspects: flow of heat for radiation, heating of the walls and hydraulic 
losses. The carried through numeric simulations had show some advantages in the case of the use of the gas as fuel: bigger efficiency; low temperatures of in the grate zone; bigger temperatures of the combustion products, that contribute for the burning fullest of the fuel.

\section{Referências}

[1] V.E. Alemassov, A.F. Dregalin e A.P. Tishin, "Propriedades Termodinâmicas e Termofísicas dos Produtos de Combustão", Guia, Vol. 1, VINITI, Moscou, 1971.

[2] C.J. Auth e R. Iskhakova, Pesquisa das propriedades termodinâmicas e termofísicas dos produtos de combustão de biomassa, em "Proceedings do VI LATCYM e VI ENCIT", Vol. 3, pp. 1769-1774, 1996.

[3] S. Dalepiane, V.G. Krioukov e L. Dalabrida, Modelo computacional de processos em fornalhas para secagem de grãos, em "Proceedings do XVIII CILAMCE", Vol. 1, pp. 541-547, 1997.

[4] J.F. Gortishev e V.V. Olimpiev, "Trocadores de Calor com Transferência de Calor Intensificada", UETK, Kazan, Rússia, 1999.

[5] B.M. Jenkins, Fuel properties for biomass materials, em "Inter. Symposium on Application and Management of the Energy in Agriculture", Indiana, 1990.

[6] R.P. Lans, L.T. Pedersen, A. Jensen, P. Glarborg e K. Dam-Johansen, Modeling and experiments of straw in a grate furnace, Biomass $\&$ Bioenergy, 19 (2000), 199-208.

[7] A. Lencastre, "Manual de Hidráulica Geral", Edgard Blücher Ltda, São Paulo, 1972.

[8] G.A. Mukhachev e V.K. Shukin, "Thermodynamics e Heat Transfer", Vischaia Schkola, Moscou, 1991.

[9] J.T.V. Pereira, G. Martins e T.P.F. Borges, Burner of wood concurrent flow with airflow - Project and test, em "Proceedings do XII Brazilian Congress of Mechanical Engineering", 1993.

[10] R. Pletka, R.C. Brown e J. Smeenk, Indirectly heated biomass gasification using a latent heat ballast. Part II: Modeling, Biomass \& Bioenergy, 20 (2001), 307-315.

[11] L.E. Sisson e D.R. Pitts, "Fenômenos de Transporte", Guanabara, Rio de Janeiro, 1988.

[12] P. Stehlik, S. Zagermann e T. Gangler, Furnace integration into processes justified by detailed calculation using a simple mathematical model, Chem. Eng. and Processing, 34 (1995), 9-23.

[13] P. Stehlik, J. Kohoutek e V. Jebacek, Simple mathematical model of furnace and its possible applications, Computers Chem. Eng., 20 (1996), 1369-1372.

[14] D.A. Tillman, A.J. Rossi e W.D. Kitto, "Wood Combustion", Academic Press Inc., New York, 203p, 1981. 